\title{
Normal forms of dispersive scalar Poisson brackets with two independent variables
}

\author{
Guido Carlet $^{1}$ - Matteo Casati ${ }^{2,3}$ (D) \\ Sergey Shadrin ${ }^{4}$
}

Received: 13 October 2017 / Revised: 16 March 2018 / Accepted: 18 March 2018 /

Published online: 26 March 2018

(C) The Author(s) 2018

\begin{abstract}
We classify the dispersive Poisson brackets with one dependent variable and two independent variables, with leading order of hydrodynamic type, up to Miura transformations. We show that, in contrast to the case of a single independent variable for which a well-known triviality result exists, the Miura equivalence classes are parametrised by an infinite number of constants, which we call numerical invariants of the brackets. We obtain explicit formulas for the first few numerical invariants.
\end{abstract}

Keywords Poisson brackets · Poisson cohomology · Hamiltonian operator · Miura transformation

\section{Mathematics Subject Classification 37K05}

\section{Contents}

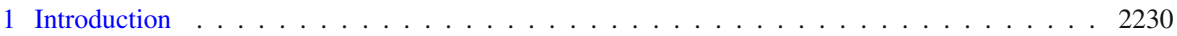

2 Theta formalism . . . . . . . . . . . . . . . . . . . . . 2233

3 Poisson cohomology . . . . . . . . . . . . . . . . . . . . . . 2235

4 Proof of the main theorem . . . . . . . . . . . . . . . . . . . . . . . . 2238

5 The numerical invariants of the Poisson bracket . . . . . . . . . . . . . . . . . . . . . . . . 2247

References . . . . . . . . . . . . . . . . . . . . . . . 2252

$凶 \quad$ Matteo Casati

M.Casati@lboro.ac.uk; matteo.casati.bg@gmail.com

1 IMB UMR 5584 CNRS Université Bourgogne Franche-Compté, 21000 Dijon, France

2 Istituto Nazionale d'Alta Matematica, Rome, Italy

3 Department of Mathematical Sciences, Loughborough University, Loughborough LE11 3TU, UK

4 Korteweg-de Vries Instituut voor Wiskunde, Universiteit van Amsterdam, Postbus 94248, 1090 GE Amsterdam, The Netherlands 


\section{Introduction}

Let $\mathcal{A}$ be the space of differential polynomials in the variable $u$, i.e. formal power series in the variables $\partial_{x^{1}}^{k_{1}} \partial_{x^{2}}^{k_{2}} u$ with coefficients which are smooth functions of $u$ :

$$
\mathcal{A}=C^{\infty}(U)\left[\left[\left\{u^{\left(k_{1}, k_{2}\right)}=\partial_{x^{1}}^{k_{1}} \partial_{x^{2}}^{k_{2}} u \text { with } k_{1}, k_{2} \geqslant 0,\left(k_{1}, k_{2}\right) \neq(0,0)\right\}\right]\right],
$$

for $U \subset \mathbb{R}$. The standard degree deg on $\mathcal{A}$ counts the number of derivatives $\partial_{x^{1}}, \partial_{x^{2}}$ in a monomial, i.e. it is defined by $\operatorname{deg}\left(\partial_{x^{1}}^{k_{1}} \partial_{x^{2}}^{k_{2}} u\right)=k_{1}+k_{2}$.

In this paper, we classify, up to Miura transformations, the dispersive Poisson brackets with one dependent variable $u$ and two independent variables $x^{1}, x^{2}$ of the form

$$
\begin{aligned}
& \left\{u\left(x^{1}, x^{2}\right), u\left(y^{1}, y^{2}\right)\right\} \\
& =\left\{u\left(x^{1}, x^{2}\right), u\left(y^{1}, y^{2}\right)\right\}^{0} \\
& \quad+\sum_{k>0} \epsilon^{k} \sum_{\substack{k_{1}, k_{2} \geqslant 0 \\
k_{1}+k_{2} \leqslant k+1}} A_{k ; k_{1}, k_{2}}(u(x)) \delta^{\left(k_{1}\right)}\left(x^{1}-y^{1}\right) \delta^{\left(k_{2}\right)}\left(x^{2}-y^{2}\right)
\end{aligned}
$$

where $A_{k ; k_{1}, k_{2}} \in \mathcal{A}$ and $\operatorname{deg} A_{k ; k_{1}, k_{2}}=k-k_{1}-k_{2}+1$.

The leading term $\left\{u\left(x^{1}, x^{2}\right), u\left(y^{1}, y^{2}\right)\right\}^{0}$ is a (scalar, two-dimensional) Poisson bracket of Dubrovin-Novikov (or hydrodynamic) type [12,13], and in other words it is of the form

$$
\begin{aligned}
& \left\{u\left(x^{1}, x^{2}\right), u\left(y^{1}, y^{2}\right)\right\}^{0} \\
& \quad=\sum_{i=1}^{2}\left[g^{i}(u(x)) \partial_{x^{i}}+b^{i}(u(x)) \partial_{x^{i}} u(x)\right] \delta\left(x^{1}-y^{1}\right) \delta\left(x^{2}-y^{2}\right),
\end{aligned}
$$

which we assume to be non-degenerate.

The conditions imposed on the functions $g^{i}(u)$ and $b^{i}(u)$ by the requirement that $\{,\}^{0}$ is skew-symmetric and satisfies the Jacobi identity have been studied by several authors $[15,21,22]$. We require the additional condition that the bracket is non-degenerate, namely that the bracket does not vanish for any value of the function $u(x)$. In the specific case considered here, where there is a single dependent variable and two independent variables, such conditions guarantee the existence of a change of coordinates in the dependent variable (a Miura transformation of the first kind), to a flat coordinate that we still denote with $u$, in which the bracket assumes the form

$$
\begin{aligned}
\left\{u\left(x^{1}, x^{2}\right), u\left(y^{1}, y^{2}\right)\right\}^{0}= & c^{1} \delta^{(1)}\left(x^{1}-y^{1}\right) \delta\left(x^{2}-y^{2}\right) \\
& +c^{2} \delta\left(x^{1}-y^{1}\right) \delta^{(1)}\left(x^{2}-y^{2}\right) .
\end{aligned}
$$


We can moreover perform (see [2]) a linear change in the independent variables $x^{1}$, $x^{2}$ such that the Poisson bracket assumes the standard form

$$
\left\{u\left(x^{1}, x^{2}\right), u\left(y^{1}, y^{2}\right)\right\}^{0}=\delta\left(x^{1}-y^{1}\right) \delta^{(1)}\left(x^{2}-y^{2}\right) .
$$

The Miura transformations (of the second kind [18]) are changes of variable of the form

$$
v=u+\sum_{k \geqslant 1} \epsilon^{k} F_{k}
$$

where $F_{k} \in \mathcal{A}$ and $\operatorname{deg} F_{k}=k$. They form a group called Miura group. We say that two Poisson brackets which are mapped to each other by a Miura transformation are Miura equivalent.

As follows from the discussion so far, the classification of dispersive Poisson brackets of the form (1) (with non-degeneracy condition) under Miura transformations (3), diffeomorphisms of the dependent variable and linear changes of the independent variables reduces to the problem of finding the normal forms of the equivalence classes under Miura transformations of the second kind (3) of the Poisson brackets (1) with leading term (2).

We solve this problem in our main result:

Theorem 1 The normal form of Poisson brackets (1) with leading term (2) under Miura transformations of the second kind is given by

$$
\begin{aligned}
\left\{u\left(x^{1}, x^{2}\right), u\left(y^{1}, y^{2}\right)\right\}= & \delta\left(x^{1}-y^{1}\right) \delta^{(1)}\left(x^{2}-y^{2}\right) \\
& +\sum_{k \geqslant 1} \epsilon^{2 k+1} c_{k} \delta^{(2 k+1)}\left(x^{1}-y^{1}\right) \delta\left(x^{2}-y^{2}\right)
\end{aligned}
$$

for a sequence of constants $c=\left(c_{1}, c_{2}, \ldots\right)$.

Remark 1 By "normal form", in the main theorem, we mean that:

i. for any choice of constants $c_{k}$ formula (4) defines a Poisson bracket which is a deformation of (2);

ii. two Poisson brackets of the form (4) are Miura equivalent if and only if they are defined by the same constants $c_{k}$;

iii. and any Poisson bracket of the form (1) can be brought to the normal form (4) by a Miura transformation.

We call the constants $c_{k}$ the numerical invariants of the Poisson bracket.

Example 1 (Hamiltonian structure of KP equation, [8]) Kadomtsev-Petviashvili (KP) equation describes two-dimensional shallow water waves, being a generalisation of $K \mathrm{~d} V$ equation. In its standard form, it is a $(2+1)$-dimensional PDE for a scalar field

$$
\partial_{x}\left(u_{t}+6 u u_{x}+u_{x x x}\right)=u_{y y}
$$


KP equation is Hamiltonian and integrable [24]; it is generally treated as the compatibility condition of an integrable $(1+1)$-dimensional hierarchy, where both the $t$ and $y$ coordinates play the role of times. However, it is possible to cast the equation in evolutionary form, with the introduction of the inverse derivative operator $\partial_{x}^{-1}$. KP equation can be written as

$$
u_{t}=\partial_{x}^{-1} u_{y y}-\partial_{x}\left(3 u^{2}+u_{x x}\right)
$$

which is Hamiltonian with respect to the Hamiltonian functional

$$
H=\int\left(\frac{\left(\partial_{x}^{-1} u_{y}\right)^{2}}{2}+\frac{u_{x}^{2}}{2}-u^{3}\right)
$$

and the Poisson bracket

$$
\{u(x, y), u(w, z)\}=\delta^{(1)}(x-w) \delta(y-z) .
$$

The Poisson bracket (5) is of the form (4) for $c_{k} \equiv 0$ and the relabeling of the independent variables $\left(x^{1} \rightarrow y, x^{2} \rightarrow x, y^{1} \rightarrow z, y^{2} \rightarrow w\right)$.

The deformation theory of Hamiltonian — and, albeit not addressed in our paper, bi-Hamiltonian-structures plays an important role in the classification of integrable Hamiltonian PDEs $[10,14]$. Most results in this field have been obtained for $(1+1)$ dimensional systems, namely the ones that depend only on one space variable.

The main result in this line of research is the triviality theorem $[9,14,16]$ of Poisson brackets of Dubrovin-Novikov type. Together with the classical results by Dubrovin and Novikov [12], this allows to conclude that the dispersive deformations of non-degenerate Dubrovin-Novikov brackets are classified by the signature of a pseudo-Riemannian metric. Similarly, deformations of bi-Hamiltonian pencils $[1,20]$ are parametrised by functions of one variable, the so-called central invariants $[10,11]$; in a few special cases, the corresponding bi-Hamiltonian cohomology has been computed, in particular for scalar brackets $[4,5,19]$, and in the semi-simple $n$-component case $[3,6]$. The $(2+1)$-dimensional case is much less studied: the classification of the structures of hydrodynamic type has been completed up to the four-component case [15], and the nontriviality of the Poisson cohomology in the two-component case has been established [7]. In our recent paper [2] we computed the Poisson cohomology for scalar-namely, one-component—brackets. Since such a cohomology is far from being trivial, the actual classification of the dispersive deformations of such brackets is a highly complicated task. We address and solve it in the present paper.

The outline of the paper is as follows: in Sect. 1 we quickly recall basic definitions and facts related with the theta formalism. In Sect. 2, we specialise some results from our previous work [2] to the $D=2$ case to obtain an explicit description of the second Poisson cohomology. In Sect. 3, we prove our main result. The proof is split into three steps corresponding to the three parts in Remark 1. In Sect. 4.4, we prove some technical lemmas that are required in the proof of Proposition 2. Finally, in Sect. 4 we give an explicit expression of the first few numerical invariants of the Poisson bracket. 


\section{Theta formalism}

We present here a short summary of the basic definitions of the theta formalism for local variational multivector fields, specialising the formulas to the scalar case with two independent variables, i.e. $N=1, D=2$. We refer the reader to [2] for the general $N, D$ case.

Let $\mathcal{A}$ be the space of differential polynomials

$$
\mathcal{A}=C^{\infty}(\mathbb{R})\left[\left[\left\{u^{(s, t)}, s, t \geqslant 0,(s, t) \neq(0,0)\right\}\right]\right]
$$

where we denote $u^{(s, t)}=\partial_{x}^{s} \partial_{y}^{t} u$, and $C^{\infty}(\mathbb{R})$ denotes the space of smooth functions in the variable $u$. The standard gradation $\operatorname{deg}$ on $\mathcal{A}$ is given by $\operatorname{deg} u^{(s, t)}=s+t$. We denote $\mathcal{A}_{d}$ the homogeneous component of degree $d$.

Using the standard derivations $\partial_{x}$ and $\partial_{y}$ on $\mathcal{A}$, we define the space of local functionals as

$$
\mathcal{F}=\frac{\mathcal{A}}{\partial_{x} \mathcal{A}+\partial_{y} \mathcal{A}}
$$

and the projection map from $\mathcal{A}$ to $\mathcal{F}$ is denoted by a double integral, which associates to $f \in \mathcal{A}$ the element

$$
\int f \mathrm{~d} x \mathrm{~d} y
$$

in $\mathcal{F}$. Moreover, we will denote by the partial integrals $\int \mathrm{d} x, \int \mathrm{d} y$ the projections from $\mathcal{A}$ to the quotient spaces $\mathcal{A} / \partial_{x} \mathcal{A}, \mathcal{A} / \partial_{y} \mathcal{A}$.

The variational derivative of a local functional $F=\int f$ is defined as

$$
\frac{\delta F}{\delta u}=\sum_{s, t \geqslant 0}\left(-\partial_{x}\right)^{s}\left(-\partial_{y}\right)^{t} \frac{\partial f}{\partial u^{(s, t)}}
$$

A local $p$-vector $P$ is a linear $p$-alternating map from $\mathcal{F}$ to itself of the form

$$
P\left(I_{1}, \ldots, I_{p}\right)=\int P_{\left(s_{1}, t_{1}\right), \ldots,\left(s_{p}, t_{p}\right)} \partial_{x}^{s_{1}} \partial_{y}^{t_{1}}\left(\frac{\delta I_{1}}{\delta u}\right) \cdots \partial_{x}^{s_{p}} \partial_{y}^{t_{p}}\left(\frac{\delta I_{p}}{\delta u}\right) \mathrm{d} x \mathrm{~d} y
$$

where $P_{\left(s_{1}, t_{1}\right), \ldots,\left(s_{p}, t_{p}\right)} \in \mathcal{A}$, for arbitrary $I_{1}, \ldots, I_{p} \in \mathcal{F}$. We denote the space of local $p$-vectors by $\Lambda^{p} \subset \operatorname{Alt}^{p}(\mathcal{F}, \mathcal{F})$.

Clearly an expression of the form (1) defines a local bivector by the usual formula

$$
\left\{I_{1}, I_{2}\right\}=\int \frac{\delta I_{1}}{\delta u\left(x^{1}, y^{1}\right)}\left\{u\left(x^{1}, y^{1}\right), u\left(x^{2}, y^{2}\right)\right\} \frac{\delta I_{2}}{\delta u\left(x^{2}, y^{2}\right)} \mathrm{d} x^{1} \mathrm{~d} y^{1} \mathrm{~d} x^{2} \mathrm{~d} y^{2}
$$


which equals to

$$
\int \sum_{k \geqslant 0} \epsilon^{k} \frac{\delta I_{1}}{\delta u(x, y)} \sum_{\substack{s, t \geqslant 0 \\ s+t \leqslant k+1}} A_{k ; s, t}(u(x)) \partial_{x}^{s} \partial_{y}^{t} \frac{\delta I_{2}}{\delta u(x, y)} \mathrm{d} x \mathrm{~d} y .
$$

The theta formalism, introduced first in the context of formal calculus of variations in [16], can be easily extended to the multidimensional setting [2] and allows to treat the local multivectors in a more algebraic fashion.

We introduce the algebra $\hat{\mathcal{A}}$ of formal power series in the commutative variables $u^{(s, t)}$ and anticommuting variables $\theta^{(s, t)}$, with coefficients given by smooth functions of $u$, i.e.

$$
\hat{\mathcal{A}}:=C^{\infty}(\mathbb{R})\left[\left[\left\{u^{(s, t)},(s, t) \neq(0,0)\right\} \cup\left\{\theta^{(s, t)}\right\}\right]\right]
$$

The standard gradation deg and the super-gradation $\operatorname{deg}_{\theta}$ of $\hat{\mathcal{A}}$ are defined by setting

$$
\operatorname{deg} u^{(s, t)}=\operatorname{deg} \theta^{(s, t)}=s+t, \quad \operatorname{deg}_{\theta} u^{(s, t)}=0, \quad \operatorname{deg}_{\theta} \theta^{(s, t)}=1 .
$$

We denote $\hat{\mathcal{A}}_{d}$, resp. $\hat{\mathcal{A}}^{p}$, the homogeneous components of standard degree $d$, resp. super-degree $p$, while $\hat{\mathcal{A}}_{d}^{p}:=\hat{\mathcal{A}}_{d} \cap \hat{\mathcal{A}}^{p}$. Clearly $\hat{\mathcal{A}}^{0}=\mathcal{A}$. The derivations $\partial_{x}$ and $\partial_{y}$ are extended to $\hat{\mathcal{A}}$ in the obvious way.

We denote by $\hat{\mathcal{F}}$ the quotient of $\hat{\mathcal{A}}$ by the subspace $\partial_{x} \hat{\mathcal{A}}+\partial_{y} \hat{\mathcal{A}}$, and by a double integral $\int \mathrm{d} x \mathrm{~d} y$ the projection map from $\hat{\mathcal{A}}$ to $\hat{\mathcal{F}}$. Since the derivations $\partial_{x}, \partial_{y}$ are homogeneous, $\hat{\mathcal{F}}$ inherits both gradations of $\hat{\mathcal{A}}$.

It turns out, see Proposition 2 in [2], that the space of local multivectors $\Lambda^{p}$ is isomorphic to $\hat{\mathcal{F}}^{p}$ for $p \neq 1$, while $\Lambda^{1}$ is isomorphic to the quotient of $\hat{\mathcal{F}}^{1}$ by the subspace of elements of the form $\int\left(k_{1} u^{(1,0)}+k_{2} u^{(0,1)}\right) \theta$ for two constants $k_{1}, k_{2}$. Moreover $\hat{\mathcal{F}}^{1}$ is isomorphic to the space $\operatorname{Der}^{\prime}(\mathcal{A})$ of derivations of $\mathcal{A}$ that commute with $\partial_{x}$ and $\partial_{y}$.

The Schouten-Nijenhuis bracket

$$
[,]: \hat{\mathcal{F}}^{p} \times \hat{\mathcal{F}}^{q} \rightarrow \hat{\mathcal{F}}^{p+q-1}
$$

is defined as

$$
[P, Q]=\int^{D}\left(\frac{\delta P}{\delta \theta} \frac{\delta Q}{\delta u}+(-1)^{p} \frac{\delta P}{\delta u} \frac{\delta Q}{\delta \theta}\right) \mathrm{d} x \mathrm{~d} y
$$

where the variational derivative with respect to $\theta$ is defined as

$$
\frac{\delta}{\delta \theta}=\sum_{s, t \geqslant 0}\left(-\partial_{x}\right)^{s}\left(-\partial_{y}\right)^{t} \frac{\delta}{\delta \theta^{(s, t)}} .
$$


It is a bilinear map that satisfies the graded symmetry

$$
[P, Q]=(-1)^{p q}[Q, P]
$$

and the graded Jacobi identity

$$
(-1)^{p r}[[P, Q], R]+(-1)^{q p}[[Q, R], P]+(-1)^{r q}[[R, P], Q]=0
$$

for arbitrary $P \in \hat{\mathcal{F}}^{p}, Q \in \hat{\mathcal{F}}^{q}$ and $r \in \hat{\mathcal{F}}^{r}$.

A bivector $P \in \hat{\mathcal{F}}^{2}$ is a Poisson structure when $[P, P]=0$. In such case $d_{P}:=$ $\operatorname{ad}_{P}=[P, \cdot]$ squares to zero, as a consequence of the graded Jacobi identity, and the cohomology of the complex $\left(\hat{\mathcal{F}}, d_{P}\right)$ is called Poisson cohomology of $P$.

The Miura transformations of the second kind [18] are changes of variable of the form

$$
u \mapsto \tilde{u}=\sum_{k=0}^{\infty} \epsilon^{k} F_{k}(u)
$$

on the space $\mathcal{A}$, where $F_{k} \in \mathcal{A}_{k}$. They form a subgroup of the general Miura group [14] which also contains the diffeomorphisms of the variable $u$. The action of a general Miura transformation of the second kind on a local multivector $Q$ in $\hat{\mathcal{F}}$ is given by the exponential of the adjoint action with respect to the Schouten-Nijenhuis bracket

$$
e^{\operatorname{ad}_{X}} Q=Q+[X, Q]+\frac{1}{2}[X,[X, Q]]+\frac{1}{6}[X,[X,[X, Q]]]+\cdots,
$$

where $X \in \hat{\mathcal{F}}_{\geqslant 1}^{1}$ is a local vector field such that $e^{\operatorname{ad}_{X}} u=\tilde{u}$.

\section{Poisson cohomology}

In our previous paper [2], we gave a description of the Poisson cohomology of a scalar multidimensional Poisson bracket in terms of the cohomology of an auxiliary complex with constant coefficients. Our aim here is to give an explicit description of a set of generators of the Poisson cohomology in the $D=2$ case, which will be used in the proof of the main theorem in the next Section.

Let us begin by recalling without proof a few results from our paper [2], specialising them to the case $D=2$.

Consider the short exact sequences of differential complexes

$$
\begin{aligned}
& 0 \rightarrow \hat{\mathcal{A}} / \mathbb{R} \stackrel{\partial_{x}}{\rightarrow} \hat{\mathcal{A}} \stackrel{\int \mathrm{d} x}{\longrightarrow} \hat{\mathcal{F}}_{1} \rightarrow 0, \\
& 0 \rightarrow \hat{\mathcal{F}}_{1} / \mathbb{R} \stackrel{\partial_{y}}{\rightarrow} \hat{\mathcal{F}}_{1} \stackrel{\int \mathrm{d} y}{\longrightarrow} \hat{\mathcal{F}} \rightarrow 0,
\end{aligned}
$$


where the differential is induced in all spaces by

$$
\Delta=\sum_{s, t \geqslant 0} \theta^{(s, t+1)} \frac{\partial}{\partial u^{(s, t)}}
$$

On $\hat{\mathcal{F}}$ such differential coincides with $\operatorname{ad}_{\mathfrak{p}_{1}}$, where $\mathfrak{p}_{1}=\frac{1}{2} \int \theta \theta^{(0,1)} \mathrm{d} x \mathrm{~d} y$.

In the long exact sequence in cohomology associated with (6), the Bockstein homomorphism vanishes; therefore,

$$
H\left(\hat{\mathcal{F}}_{1}\right)=\frac{H(\hat{\mathcal{A}})}{\partial_{x} H(\hat{\mathcal{A}})}
$$

Moreover, the cohomology classes in $H(\hat{\mathcal{A}})$ can be uniquely represented by elements of the polynomial ring $\Theta$ generated by the anticommuting variables $\theta^{(s, 0)}, s \geqslant 0$ with real coefficients.

The map induced in cohomology by the map $\partial_{y}$ in the short exact sequence (7) vanishes; therefore, we get the following exact sequence

$$
0 \rightarrow\left(\frac{\Theta}{\partial_{x} \Theta}\right)_{d}^{p} \stackrel{\int \mathrm{d} y}{\longrightarrow} H_{d}^{p}(\hat{\mathcal{F}}) \rightarrow\left(\frac{\Theta}{\partial_{x} \Theta}\right)_{d}^{p+1} \rightarrow 0
$$

where the third arrow is the Bockstein homomorphism.

This sequence allows us to write the Poisson cohomology $H^{p}(\hat{\mathcal{F}})$ as a sum of two homogeneous subspaces of $\Theta / \partial_{x} \Theta$ in super-degree $p$ and $p+1$, respectively, where the first one is simply injected, while the second one has to be reconstructed via the inverse to the Bockstein homomorphism.

Let $\iint a \mathrm{~d} x \mathrm{~d} y \in \hat{\mathcal{F}}_{d}^{p}$ be an $\operatorname{ad}_{\mathfrak{p}_{1}}$-cocycle. Then, there exist $b, b^{\prime} \in \hat{\mathcal{A}}_{d}^{p+1}$ such that

$$
\Delta a=\partial_{y} b+\partial_{x} b^{\prime}
$$

The Bockstein homomorphism assigns to the cocycle $\iint a \mathrm{~d} x \mathrm{~d} y$ the cocycle $\int b \mathrm{~d} x \in$ $\hat{\mathcal{F}}_{d}^{p+1}$.

Let us define a map $\mathcal{B}: \Theta \rightarrow \hat{\mathcal{A}}$ by

$$
\mathcal{B}=\sum_{i \geqslant 0} u^{(i, 0)} \frac{\partial}{\partial \theta^{(i, 0)}}
$$

which clearly commutes with $\partial_{x}$, and therefore induces a map from $\frac{\Theta}{\partial_{x} \Theta}$ to $\hat{\mathcal{F}}$. We have that

$$
\Delta \mathcal{B}=\partial_{y},
$$


and consequently, $\mathcal{B}$ defines a splitting map

$$
\mathcal{B}:\left(\frac{\Theta}{\partial_{x} \Theta}\right)_{d}^{p+1} \rightarrow H_{d}^{p}(\hat{\mathcal{F}})
$$

for the short exact sequence (8).

We have therefore shown that

Lemma $1 H_{d}^{p}(\hat{\mathcal{F}})=\left(\frac{\Theta}{\partial_{x} \Theta}\right)_{d}^{p} \oplus \mathcal{B}\left(\frac{\Theta}{\partial_{x} \Theta}\right)_{d}^{p+1}$.

We remark that this lemma gives an explicit description of representatives of the cohomology classes in $H_{d}^{p}(\hat{\mathcal{F}})$. In particular, the only non-trivial classes in $\Theta / \partial_{x} \Theta$ in super-degree $p=2$ are given by $\theta \theta^{(2 k+1,0)}$ for $k \geqslant 1$ and correspond to the deformations of the Poisson brackets in Theorem 1. The following reformulation of this observation will be useful in the proof of Proposition 2:

\section{Corollary 1}

$$
\begin{gathered}
H_{2 k}^{2}(\hat{\mathcal{F}})=\mathcal{B}\left(\frac{\Theta}{\partial_{x} \Theta}\right)_{2 k}^{3}, \\
H_{2 k+1}^{2}(\hat{\mathcal{F}})=\mathbb{R} \theta \theta^{(2 k+1,0)} \oplus \mathcal{B}\left(\frac{\Theta}{\partial_{x} \Theta}\right)_{2 k+1}^{3}
\end{gathered}
$$

Moreover, we can define an explicit basis of $\left(\frac{\Theta}{\partial_{x} \Theta}\right)_{d}^{3}$ and $\mathcal{B}\left(\frac{\Theta}{\partial_{x} \Theta}\right)_{d}^{3}$ :

Lemma 2 A basis of $\left(\frac{\Theta}{\partial_{x} \Theta}\right)_{d}^{3}$ is given by representatives

$$
\begin{aligned}
& \theta^{k-l} \theta^{k-l-1} \theta^{2 l}, \quad l=0, \ldots,\left\lfloor\frac{k-2}{3}\right\rfloor, \quad \text { for } d=2 k-1, \\
& \theta^{k-l} \theta^{k-l-1} \theta^{2 l+1}, \quad l=0, \ldots,\left\lfloor\frac{k-3}{3}\right\rfloor, \quad \text { for } d=2 k,
\end{aligned}
$$

where we use the notation $\theta^{k}=\theta^{(k, 0)}$.

Proof More generally we can prove that a basis of $\left(\frac{\Theta}{\partial_{x} \Theta}\right)_{d}^{p}$ is given by

$$
\theta^{i_{2}+1} \theta^{i_{2}} \theta^{i_{3}} \cdots \theta^{i_{p}}
$$

with

$$
i_{2}>i_{3}>\cdots>i_{p} \geqslant 0, \quad 1+2 i_{2}+i_{3}+\cdots+i_{p}=d .
$$

A basis of $\Theta_{d}^{p}$ is given by monomials $\theta^{i_{1}} \ldots \theta^{i_{p}}$ with

$$
i_{1}>i_{2}>\cdots>i_{p} \geqslant 0, \quad i_{1}+\cdots+i_{p}=d .
$$


We arrange such monomials in lexicographic order, that is, we say that $\theta^{i_{1}} \cdots \theta^{i_{p}}>$ $\theta^{j_{1}} \cdots \theta^{j_{p}}$ if $i_{1}>j_{1}$, or if $i_{1}=j_{1}$ and $i_{2}>j_{2}$, and so on.

For an element $a=\theta^{i_{1}} \cdots \theta^{i_{p}}$ of the basis of $\Theta_{d-1}^{p}$, we have that the leading term (in lexicographic order) of $\partial_{x} a$ is given by

$$
\left(\partial_{x} a\right)^{\text {top }}=\theta^{i_{1}+1} \theta^{i_{2}} \cdots \theta^{i_{p}} .
$$

Note that if $a_{1}>a_{2}$, then $\left(\partial_{x} a_{1}\right)^{\text {top }}>\left(\partial_{x} a_{2}\right)^{\text {top }}$. This implies that the images $\partial_{x} a$ of the monomials $a \in \Theta_{d-1}^{p}$ are linearly independent in $\Theta_{d}^{p}$. Given a representative of a class in $\left(\frac{\Theta}{\partial_{x} \Theta}\right)_{d}^{p}$, we can express all the monomials of the form (11) in terms of combinations of monomials of strictly lower lexicographic order. It follows that a basis can be chosen in the form (10).

By specialising to the case $p=3$, and spelling out the allowed sets of indexes, we obtain the statement of the lemma.

It follows that a basis of $\mathcal{B}\left(\frac{\Theta}{\partial_{x} \Theta}\right)_{d}^{3}$ is given by the elements

$$
\mathcal{B}\left(\theta^{(a, 0)} \theta^{(b, 0)} \theta^{(c, 0)}\right)=u^{(a, 0)} \theta^{(b, 0)} \theta^{(c, 0)}-u^{(b, 0)} \theta^{(a, 0)} \theta^{(c, 0)}+u^{(c, 0)} \theta^{(a, 0)} \theta^{(b, 0)},
$$

for indices $a, b, c$ chosen as in the basis above.

\section{Proof of the main theorem}

Let us first reformulate our main statement in the $\theta$-formalism.

The Poisson bracket of Dubrovin-Novikov type of the form (2) corresponds to the element

$$
\mathfrak{p}_{1}=\frac{1}{2} \iint \theta \theta^{(0,1)} \mathrm{d} x \mathrm{~d} y
$$

in $\hat{\mathcal{F}}_{1}^{2}$. The bivector $\delta^{(2 k+1)}\left(x^{1}-y^{1}\right) \delta\left(x^{2}-y^{2}\right)$ corresponds to the element in $\hat{\mathcal{F}}_{2 k+1}^{2}$ given by

$$
\mathfrak{p}_{2 k+1}=\frac{1}{2} \iint \theta \theta^{(2 k+1,0)} \mathrm{d} x \mathrm{~d} y \text {. }
$$

Therefore, the normal form (4) in $\theta$-formalism corresponds to the element

$$
\mathfrak{p}(c)=\mathfrak{p}_{1}+\sum_{k \geqslant 1} c_{k} \mathfrak{p}_{2 k+1}
$$

in $\hat{\mathcal{F}}^{2}$.

The proof of Theorem 1 reduces to prove the three statements listed in Remark 1. 


\subsection{Proof of the statement in Remark 1.i}

Our first observation is:

Lemma 3 The bivectors $\mathfrak{p}_{2 k+1}$ with $k \geqslant 0$ are pairwise compatible, i.e.

$$
\left[\mathfrak{p}_{2 n+1}, \mathfrak{p}_{2 m+1}\right]=0, \quad n, m \geqslant 0 .
$$

Proof The Poisson bivectors $\mathfrak{p}_{k}$ do not depend on $u$ and its derivatives; therefore, the variational derivatives w.r.t. $u$ appearing in the definition of Schouten-Nijenhuis bracket are vanishing.

It clearly follows that $\mathfrak{p}(c)$ is a Poisson bivector for any choice of the constants $c=\left(c_{1}, c_{2}, \ldots\right)$.

\subsection{Proof of the statement in Remark 1.ii}

Next we show that for any distinct choice of the constants $c=\left(c_{1}, c_{2}, \ldots\right)$ the corresponding bivector $P$ belongs to a different equivalence class under Miura transformations.

Proposition 1 Let $\mathfrak{p}(c)$, resp. $\mathfrak{p}(\tilde{c})$, be the Poisson bivector of the normal form (13) corresponding to a choice $c=\left(c_{1}, c_{2}, \ldots\right)$, resp. $\tilde{c}=\left(\tilde{c}_{1}, \tilde{c}_{2}, \ldots\right)$, of constants. If the two sequences $c$ and $\tilde{c}$ are not identically equal, then there is no Miura transformation of the second kind which maps $\mathfrak{p}(c)$ to $\mathfrak{p}(\tilde{c})$.

Proof Assume there is a Miura transformation of the second kind mapping $\mathfrak{p}(c)$ to $\mathfrak{p}(\tilde{c})$, i.e.

$$
e^{\operatorname{ad}_{X}} \mathfrak{p}(c)=\mathfrak{p}(\tilde{c}),
$$

for $X \in \hat{\mathcal{F}}_{\geqslant 1}^{1}$. This identity can be rewritten as

$$
\left(\frac{e^{\operatorname{ad}_{X}}-1}{\operatorname{ad}_{X}}\right) \operatorname{ad}_{X} \mathfrak{p}(c)=\mathfrak{p}(\tilde{c})-\mathfrak{p}(c) .
$$

The operator inside the brackets has the form

$$
\left(\frac{e^{\operatorname{ad}_{X}}-1}{\operatorname{ad}_{X}}\right)=1+\frac{1}{2} \operatorname{ad}_{X}+\cdots
$$

and therefore we can invert it. We obtain

$$
\operatorname{ad}_{X} \mathfrak{p}(c)=\left(\frac{e^{\operatorname{ad}_{X}}-1}{\operatorname{ad}_{X}}\right)^{-1}(\mathfrak{p}(\tilde{c})-\mathfrak{p}(c))
$$


By assumption the two sequences, $c$ and $\tilde{c}$ are not identically equal, and hence there exists a smallest index $k$ for which $c_{k} \neq \tilde{c}_{k}$. It follows that

$$
\mathfrak{p}(\tilde{c})-\mathfrak{p}(c)=\left(\tilde{c}_{k}-c_{k}\right) \mathfrak{p}_{2 k+1}+\cdots,
$$

where the dots denote terms of standard degree greater than $2 k+1$. We conclude that $\operatorname{ad}_{X} \mathfrak{p}(c)$ has to vanish in standard degree less or equal to $2 k$, i.e.

$$
\left(\operatorname{ad}_{X} \mathfrak{p}(c)\right) \leqslant 2 k=0
$$

So, the leading order term in the standard degree in (14) is

$$
\left(\operatorname{ad}_{X} \mathfrak{p}(c)\right)_{2 k+1}=\left(\tilde{c}_{k}-c_{k}\right) \mathfrak{p}_{2 k+1} .
$$

The key point of the proof is to prove that the lefthand side is a $\operatorname{ad}_{\mathfrak{p}_{1}}$ coboundary, which leads to a contradiction since we know that $\mathfrak{p}_{2 k+1}$ is a non-trivial class in $H_{2 k+1}^{2}\left(\hat{\mathcal{F}}, \mathfrak{p}_{1}\right)$.

Notice that the lefthand side in (16) can be written

$$
\operatorname{ad}_{\mathfrak{p}_{1}} X_{2 k}+\sum_{s=1}^{k-1} c_{s} \operatorname{ad}_{\mathfrak{p}_{2 s+1}} X_{2(k-s)}=\left(\tilde{c}_{k}-c_{k}\right) \mathfrak{p}_{2 k+1}
$$

and hence it is sufficient to prove that the sum in the lefthand side is in the image of $\operatorname{ad}_{\mathfrak{p}_{1}}$.

Equation (15) gives a sequence of constraints on $X$. Let us consider in particular the constraints with odd degree

$$
\left(\operatorname{ad}_{X} \mathfrak{p}(c)\right)_{2 s+1}=0, \quad s=1, \ldots, k-1,
$$

which can be written

$$
\operatorname{ad}_{\mathfrak{p}_{1}} X_{2 s}+\sum_{l=1}^{s-1} c_{l} \operatorname{ad}_{\mathfrak{p}_{2 l+1}} X_{2(s-l)}=0
$$

This equation for $s=1$ simply says that $X_{2}$ is a cocycle w.r.t. $\operatorname{ad}_{\mathfrak{p}_{1}}$,

$$
\operatorname{ad}_{\mathfrak{p}_{1}} X_{2}=0
$$

By the vanishing of the Poisson cohomology $H_{2}^{1}\left(\hat{\mathcal{F}}, \mathfrak{p}_{1}\right), X_{2}$ is necessarily a coboundary, i.e.

$$
X_{2}=\operatorname{ad}_{\mathfrak{p}_{1}} f_{1}
$$

for some $f_{1} \in \hat{\mathcal{F}}_{1}^{0}$. 
More generally, we have that for each $s=1, \ldots, k-1$

$$
X_{2 s}=\operatorname{ad}_{\mathfrak{p}_{1}} f_{2 s-1}+\sum_{l=1}^{s-1} c_{l} \operatorname{ad}_{\mathfrak{p}_{2 l+1}} f_{2(s-l)-1}
$$

for some $f_{2 l-1} \in \hat{\mathcal{F}}_{2 l-1}^{0}, l=1, \ldots, 2 s-1$. We can prove this by induction. Let us therefore assume that (19) holds for $s=1, \ldots, t-1$ for $t \leqslant k-1$, and show that it holds for $s=t$ too. Substituting the inductive assumption in (18) for $s=t$, we get that

$$
\operatorname{ad}_{\mathfrak{p}_{1}}\left(X_{2 t}-\sum_{l=1}^{t-1} c_{l} \operatorname{ad}_{\mathfrak{p}_{2 l+1}} f_{2(t-l)-1}\right)=0
$$

The expression inside the brackets is therefore a cocycle, which has to be a coboundary due to the triviality of $H_{2 t}^{1}\left(\hat{\mathcal{F}}, \mathfrak{p}_{1}\right)$, i.e.

$$
X_{2 t}-\sum_{l=1}^{t-1} c_{l} \operatorname{ad}_{\mathfrak{p}_{2 l+1}} f_{2(t-l)-1}=\operatorname{ad}_{\mathfrak{p}_{1}} f_{2 t-1},
$$

for some $f_{2 t-1} \in \hat{\mathcal{F}}_{2 t-1}^{0}$. This gives (19) for $s=t$.

Substituting (19) in (17), we get that

$$
\left(\tilde{c}_{k}-c_{k}\right) \mathfrak{p}_{2 k+1}=\operatorname{ad}_{\mathfrak{p}_{1}}\left(X_{2 k}-\sum_{s=1}^{k-1} c_{s} \operatorname{ad}_{\mathfrak{p}_{2 s+1}} f_{2(k-s)-1}\right),
$$

up to a term that can be written as

$$
\sum_{n \geqslant 2}^{k-1}\left[\sum_{\substack{s, l \geqslant 1 \\ s+l=n}} c_{s} c_{l} \operatorname{ad}_{\mathfrak{p}_{2 s+1}} \operatorname{ad}_{\mathfrak{p}_{2 l+1}}\right] f_{2(k-n)-1}
$$

and therefore clearly vanishes. Equation (20) leads to sought contradiction.

The Lemma is proved.

\subsection{Proof of the statement in Remark 1.iii}

Finally, we prove that any Poisson bivector with leading order $\mathfrak{p}_{1}$ given by (12) can always be brought to the form (13) by a Miura transformation of the second kind.

Proposition 2 Let $P \in \hat{\mathcal{F}}_{\geqslant 1}^{2}$ be a Poisson bivector with degree one term equal to $\mathfrak{p}_{1}$. Then there is a Miura transformation that maps $P$ to a $\mathfrak{p}(c)$ for a choice of constants $c=\left(c_{1}, c_{2}, \ldots\right)$. 
Proof The Poisson bivector $P \in \hat{\mathcal{F}}_{\geqslant 1}^{2}$ has to satisfy $[P, P]=0$. We want to show by induction that, taking into account this equation, it is possible, by repeated application of Miura transformations, to put all terms in normal form and to remove all terms that come from the Bockstein homomorphism.

Let us denote by $\mathfrak{p}_{(s)}\left(c_{1}, \ldots, c_{\lfloor s / 2-1\rfloor}\right)$ a bivector of the form

$$
\begin{aligned}
& \mathfrak{p}_{(2 k)}\left(c_{1}, \ldots, c_{k-1}\right)=\mathfrak{p}_{1}+\sum_{l=1}^{k-1} c_{l} \mathfrak{p}_{2 l+1}+\sum_{l=k+1}^{2 k-1} Q_{l}+P_{2 k}+\cdots \\
& \mathfrak{p}_{(2 k+1)}\left(c_{1}, \ldots, c_{k-1}\right)=\mathfrak{p}_{1}+\sum_{l=1}^{k-1} c_{l} \mathfrak{p}_{2 l+1}+\sum_{l=k+1}^{2 k} Q_{l}+P_{2 k+1}+\cdots
\end{aligned}
$$

for $s$, respectively, even or odd, where $Q_{l} \in \mathcal{B}\left(\frac{\Theta}{\partial_{x} \Theta}\right)_{l}^{3}, P_{l} \in \hat{\mathcal{F}}_{l}^{2}$, the dots denote higher-order terms, and

$$
\left[\mathfrak{p}_{(s)}, \mathfrak{p}_{(s)}\right]=0
$$

The inductive hypothesis is valid for $s=2$; indeed, $\mathfrak{p}_{(2)}$ is exactly of the required form.

Let us now show that by a Miura transformation a Poisson bivector of the form $\mathfrak{p}_{(s)}$ can be made of the form $\mathfrak{p}_{(s+1)}$.

When $s=2 k$ is even, in degree $2 k+1$, Eq. (21) gives

$$
\left[\mathfrak{p}_{1}, P_{2 k}\right]+\sum_{\substack{2 l+m=2 k \\ 1 \leqslant l \leqslant k-1 \\ k+1 \leqslant m \leqslant 2 k-1}}\left[c_{l} \mathfrak{p}_{2 l+1}, Q_{m}\right]=0 .
$$

The first observation is that both terms above need to be separately zero. This follows from the fact that the first term has nonzero degree in the number of derivatives w.r.t. $y$, while the second term has degree zero.

By Corollary 1 , the cohomology $H_{2 k}^{2}(\hat{\mathcal{F}})$ is given only by elements coming from the Bockstein homomorphism, and therefore exists $Q_{2 k} \in \mathcal{B}\left(\frac{\Theta}{\partial_{x} \Theta}\right)_{2 k}^{3}$ such that $P_{2 k}+$ $\operatorname{ad}_{\mathfrak{p}_{1}} X_{2 k-1}=Q_{2 k}$ for some $X_{2 k-1} \in \hat{\mathcal{F}}_{2 k-1}^{1}$.

Acting with the Miura transformation $e^{\operatorname{ad}_{X_{2 k-1}}}$ on $\mathfrak{p}_{(2 k)}$, we get a new Poisson bivector, where the terms of degree less or equal to $2 k-1$ are unchanged, the term $P_{2 k}$ has been replaced with the term $Q_{2 k}$, and the terms of higher order are in general different. We have therefore that $\mathfrak{p}_{(2 k+1)}=e^{\mathrm{ad}_{X_{2 k-1}}} \mathfrak{p}_{(2 k)}$ is of the form above, as required.

When $s=2 k+1$ is odd, in degree $2 k+2$ from (21) we get

$$
\left[\mathfrak{p}_{1}, P_{2 k+1}\right]+\sum_{\substack{2 l+m=2 k+1 \\ 1 \leqslant l \leqslant k-1 \\ k+1 \leqslant m \leqslant 2 k}}\left[c_{l} \mathfrak{p}_{2 l+1}, Q_{m}\right]+\frac{1}{2}\left[Q_{k+1}, Q_{k+1}\right]=0
$$


As in the previous case, the first term has to vanish; hence, $P_{2 k+1}$ is an $\operatorname{ad}_{\mathfrak{p}_{1}}$-cocycle. The cohomology $H_{2 k+1}^{2}(\hat{\mathcal{F}})$ decomposes in two parts; therefore, there is a constant $c_{k}$ and an element $Q_{2 k+1}$ in $\mathcal{B}\left(\frac{\Theta}{\partial_{x} \Theta}\right)_{2 k+1}^{3}$ such that $P_{2 k+1}+\operatorname{ad}_{\mathfrak{p}_{1}} X_{2 k}=c_{k} \mathfrak{p}_{2 k+1}+Q_{2 k+1}$ for some $X_{2 k} \in \hat{\mathcal{F}}_{2 k}^{1}$.

The second and third terms in (22) have also to be both zero. This follows from the fact that they have different degree in the number of $u^{(s, t)}$. As we have seen in Sect. 3, the elements $Q_{k}$ are linear in the variables $u^{(s, t)}$, while the elements $\mathfrak{p}_{k}$ do not contain them.

From the vanishing of the last term, $\left[Q_{k+1}, Q_{k+1}\right]=0$, we finally derive that $Q_{k+1}$ is zero. This is guaranteed by Lemma 4 . The proof of this Lemma, being quite technical, is given in Sect. 4.4.

Taking into account this vanishing, the action of the Miura transformation $e^{\operatorname{ad}_{X_{2 k}}}$ on $\mathfrak{p}_{(2 k+1)}$ gives exactly the term $\mathfrak{p}_{(2 k+2)}$.

By induction, we see that we can continue this procedure indefinitely; therefore, we conclude that we cannot have any non-trivial deformation coming from $\left(\frac{\Theta}{\partial_{x} \Theta}\right)^{3}$ via the Bockstein homomorphism, and that the Miura transformation $\cdots e^{\operatorname{ad}_{X_{2}}} e^{\operatorname{ad}_{X_{1}}}$ given by the composition of the Mira transformations defined above, sends the original Poisson bivector $P=\mathfrak{p}_{1}+\cdots$ to a Poisson bivector of the form $\mathfrak{p}(c)$ for a choice of constants $c_{1}, c_{2}, \ldots$.

The Proposition is proved.

\subsection{Some technical lemmas}

In this section, we prove the following statement, which is essential in the proof of Proposition 2:

Lemma 4 Let $\chi \in\left(\frac{\Theta}{\partial_{x} \Theta}\right)_{d}^{3}$ and $\mathcal{B}(\chi)$ its image through the map (9) in $\hat{\mathcal{F}}_{d}^{2}$. If $[\mathcal{B}(\chi), \mathcal{B}(\chi)]=0$, then $\chi=0$.

Proof We have

$$
\begin{aligned}
{[\mathcal{B}(\chi), \mathcal{B}(\chi)] } & =2 \iint \frac{\delta \mathcal{B}(\chi)}{\delta \theta} \frac{\delta \mathcal{B}(\chi)}{\delta u}=2 \iint \frac{\delta \mathcal{B}(\chi)}{\delta \theta} \frac{\delta \chi}{\delta \theta} \\
& =-2 \iint \mathcal{B}\left(\frac{\delta \chi}{\delta \theta}\right) \frac{\delta \chi}{\delta \theta}=-\iint \mathcal{B}\left(\frac{\delta \chi}{\delta \theta}\right)^{2},
\end{aligned}
$$

where the second and third equalities follow from the simple identities

$$
\frac{\delta \mathcal{B}(\chi)}{\delta u}=\frac{\delta \chi}{\delta \theta}, \quad\left[\mathcal{B}, \frac{\delta}{\delta \theta}\right]_{+}=0 .
$$


Since we proved that the map

$$
\mathcal{B}:\left(\frac{\Theta}{\partial_{x} \Theta}\right)_{d}^{p+1} \rightarrow H_{d}^{p}(\hat{\mathcal{F}})
$$

is injective, the vanishing of (23) implies that $\left(\frac{\delta \chi}{\delta \theta}\right)^{2}=0$ in $\left(\frac{\Theta}{\partial_{x} \Theta}\right)^{4}$. From this fact, it follows that $\chi=0$, as we prove in the remaining part of this section. ${ }^{1}$

Let sq: $\Theta_{k}^{2} \rightarrow \Theta_{2 k}^{4}$ be the map that sends an element $\alpha \in \Theta_{k}^{2}$ to $\alpha^{2} \in \Theta_{2 k}^{4}$. In the rest of this section, we will use the notation $\theta^{d}=\theta^{(d, 0)}$.

Lemma 5 The intersection of $\operatorname{sq}\left(\Theta_{k}^{2}\right)$ and $\partial_{x} \Theta_{2 k-1}^{4}$ is equal to zero. In other words, if $\alpha \in \Theta_{k}^{2}$ and $\alpha^{2}$ is $\partial_{x}$-exact, then $\alpha^{2}=0$ and, therefore, $\alpha$ is proportional to a monomial $\theta^{i} \theta^{k-i}$ for some $i=1, \ldots,\left\lfloor\frac{k-1}{2}\right\rfloor$.

Proof A basis in $\Theta_{2 k-1}^{4}$ is given by standard monomials $\theta^{i_{1}} \theta^{i_{2}} \theta^{i_{3}} \theta^{i_{4}}$ with total degree $i_{1}+i_{2}+i_{3}+i_{4}=2 k-1$. By standard monomial, we indicate a monomial where the indices are ordered as $i_{1}>i_{2}>i_{3}>i_{4} \geqslant 0$ to avoid duplicates.

We can write $\Theta_{2 k-1}^{4}=\mathcal{V}_{1} \oplus \mathcal{V}_{2}$, where a basis for $\mathcal{V}_{1}$ is given by standard monomials with the restriction $i_{1}+i_{4} \leqslant k-1$, and a basis for $\mathcal{V}_{2}$ is given by standard monomials with $i_{1}+i_{4} \geqslant k$.

It is convenient to define also the subspace $\mathcal{W}$ of $\Theta_{2 k}^{4}$ which is spanned by all monomials that appear in the $\partial_{x} \mathcal{V}_{1}$; more explicitly $\mathcal{W}$ is generated by the monomials

$$
\theta^{i_{1}+1} \theta^{i_{2}} \theta^{i_{3}} \theta^{i_{4}}, \quad \theta^{i_{1}} \theta^{i_{2}+1} \theta^{i_{3}} \theta^{i_{4}}, \quad \theta^{i_{1}} \theta^{i_{2}} \theta^{i_{3}+1} \theta^{i_{4}}, \quad \theta^{i_{1}} \theta^{i_{2}} \theta^{i_{3}} \theta^{i_{4}+1},
$$

with $i_{1}>i_{2}>i_{3}>i_{4} \geqslant 0, i_{1}+i_{4} \leqslant k-1$, and $i_{1}+i_{2}+i_{3}+i_{4}=2 k-1$.

We denote by $\Theta_{k}^{2} \cdot \Theta_{k}^{2}$ the subspace of $\Theta_{2 k}^{4}$ spanned by standard monomials $\theta^{i_{1}} \theta^{i_{2}} \theta^{i_{3}} \theta^{i_{4}}$ with $i_{1}>i_{2}>i_{3}>i_{4} \geqslant 0$ and $i_{1}+i_{2}+i_{3}+i_{4}=2 k$ with $i_{1}+i_{4}=k$ and $i_{2}+i_{3}=k$. It is indeed the subspace given by the product of two arbitrary elements of $\Theta_{k}^{2}$.

Clearly, both $\partial_{x} \mathcal{V}_{1}$ and $\Theta_{k}^{2} \cdot \Theta_{k}^{2}$ are subspaces of $\mathcal{W}$.

Let us now prove that $\partial_{x} \mathcal{V}_{2}$ has zero intersection with $\mathcal{W}$. Let $v=\sum_{\gamma} v_{\gamma} \gamma$ be an element in $\mathcal{V}_{2}$, where $\gamma$ is in the standard basis of $\mathcal{V}_{2}$ described above. Let $\partial_{x} v=$ $\sum_{\gamma} v_{\gamma} \partial_{x} \gamma \in \mathcal{W}$. We have already seen that the elements $\partial_{x} \gamma$ are linearly independent. If $\gamma=\theta^{i_{1}} \theta^{i_{2}} \theta^{i_{3}} \theta^{i_{4}}$ then $\partial_{x} \gamma$ is equal to $\theta^{i_{1}+1} \theta^{i_{2}} \theta^{i_{3}} \theta^{i_{4}}$ plus lexicographically lower terms. The lexicographically leading order term is therefore of a standard monomial $\theta^{j_{1}} \theta^{j_{2}} \theta^{j_{3}} \theta^{j_{4}}$ with $j_{1}+j_{4} \geqslant k+1$. But all basis elements in $\mathcal{W}$ are standard monomials with $j_{1}+j_{4} \leqslant k$. It follows that, if $\gamma$ is the lexicographically highest term in $v$, we must have $v_{\gamma}=0$. By induction $v$ vanishes.

\footnotetext{
1 Notice that this fact, in the case of standard differential polynomials in commuting variables, follows from a simple observation: the derivative in $x$ of a differential polynomial cannot be a square, since it has to be linear in the highest derivative. In the case of anticommuting variables, however, a quite involved proof is necessary.
} 
The two facts $\partial_{x} \mathcal{V}_{1} \subseteq \mathcal{W}$ and $\partial_{x} \mathcal{V}_{2} \cap \mathcal{W}=(0)$ imply at once that the preimage $\partial_{x}^{-1}(\mathcal{W})$ in $\Theta_{2 k-1}^{4}$ is contained in $\mathcal{V}_{1}$, and the same holds for $\Theta_{k}^{2} \cdot \Theta_{k}^{2}$ since it is a subspace of $\mathcal{W}$, i.e. we have

$$
\partial_{x}^{-1}\left(\Theta_{k}^{2} \cdot \Theta_{k}^{2}\right) \subseteq \mathcal{V}_{1}
$$

Since $\mathrm{sq}\left(\Theta_{k}^{2}\right) \subseteq \Theta_{k}^{2} \cdot \Theta_{k}^{2}$, our original problem reduces to finding the intersection of $\operatorname{sq}\left(\Theta_{k}^{2}\right)$ and $\partial_{x} \mathcal{V}_{1}$.

Let $\alpha=\sum_{i=\left\lceil\frac{k+1}{2}\right\rceil}^{k} \alpha_{i} \theta^{i} \theta^{k-i}$ be an element of $\Theta_{k}^{2}$ whose square is in $\partial_{x} \mathcal{V}_{1}$. We want to show that at most one of the coefficients $\alpha_{i}$ is nonzero. We therefore assume that at least two such coefficients are nonzero and show that it leads to a contradiction. Let $s$ be the higher index for which $\alpha_{s} \neq 0$ and $t<s$ the second higher index for which $\alpha_{t} \neq 0$.

Denote by $\mathcal{W}^{(j)}$ the subspace of $\Theta_{k}^{2} \cdot \Theta_{k}^{2}$ spanned by monomials of the form

$$
\theta^{i} \theta^{j} \theta^{k-j} \theta^{k-i} \text { for } i=k, \ldots, j+1
$$

and denote by $\widetilde{\mathcal{W}}$ the space spanned by the basis monomials in $\mathcal{W}$ which are not in $\Theta_{k}^{2} \cdot \Theta_{k}^{2}$. Notice that

$$
\Theta_{k}^{2} \cdot \Theta_{k}^{2}=\bigoplus_{j=\left\lceil\frac{k+1}{2}\right\rceil}^{k-1} \mathcal{W}^{(j)}
$$

and consequently

$$
\mathcal{W}=\tilde{\mathcal{W}} \oplus \bigoplus_{j=\left\lceil\frac{k+1}{2}\right\rceil}^{k-1} \mathcal{W}^{(j)}
$$

Observe that a monomial $\theta^{i} \theta^{j} \theta^{k-j} \theta^{k-i}$ in $\mathcal{W}^{(j)}$ can appear in the $\partial_{x}$-image of four different monomials in $\Theta_{2 k-1}^{4}$ but only two of them are elements of $\mathcal{V}_{1}$, i.e.

$$
\theta^{i-1} \theta^{j} \theta^{k-j} \theta^{k-i}, \quad \theta^{i} \theta^{j} \theta^{k-j} \theta^{k-i-1},
$$

so we only need to consider these two.

Notice that a monomial in $\mathcal{V}_{1}$ of such form, i.e. $\theta^{l} \theta^{j} \theta^{k-j} \theta^{k-l-1}$ is mapped by $\partial_{x}$ to the sum of four monomials, two of which are in $\mathcal{W}^{(j)}$, i.e.

$$
\theta^{l+1} \theta^{j} \theta^{k-j} \theta^{k-l-1}, \quad \theta^{l} \theta^{j} \theta^{k-j} \theta^{k-l},
$$

and two are in $\tilde{\mathcal{W}}$.

Since $\alpha^{2} \in \Theta_{k}^{2} \cdot \Theta_{k}^{2}$, it can be decomposed in its components $\left(\alpha^{2}\right)_{j} \in \mathcal{W}^{(j)}$, and we have in particular that 


$$
\left(\alpha^{2}\right)_{t}=2 \alpha_{s} \alpha_{t} \theta^{s} \theta^{t} \theta^{k-t} \theta^{k-s},
$$

since we have assumed that $\alpha_{i}=0$ for $i>s$ and $t<i<s$.

All these observations imply that there must be an element $\beta$ of $\mathcal{V}_{1}$ of the form

$$
\beta=\sum_{i=k-1}^{t+1} \beta_{i} \theta^{i} \theta^{t} \theta^{k-t} \theta^{k-i-1}
$$

such that its image through $\partial_{x}$ gives $\left(\alpha^{2}\right)_{t}$ plus some element in $\widetilde{\mathcal{W}}$.

The lexicographically higher term in $\beta$, i.e. for $i=k-1$, is sent by $\partial_{x}$ to a term proportional to $\theta^{k} \theta^{t} \theta^{k-t} \theta^{0}$, which does not appear in $\left(\alpha^{2}\right)_{t}$, therefore $\beta_{k-1}=0$. Proceeding like this, we set to zero all the constants $\beta_{k-1}, \ldots, \beta_{s}$. Similarly, we can proceed from the lower part of the chain and set to zero all the remaining constants $\beta_{t+1}, \ldots, \beta_{s-1}$. But then $\beta=0$, therefore $\alpha_{s} \alpha_{t}=0$ and we are led to a contradiction.

We have proved that at most one of the constants $\alpha_{i}$ can be nonzero. In such case, $\alpha^{2}=0$. The Lemma is proved.

Lemma 6 Consider an arbitrary element $\chi \in \Theta_{d}^{3}$. If $\frac{\delta \chi}{\delta \theta}=c \cdot \theta^{i} \theta^{d-i}$ for some $i=0,1, \ldots,\left\lfloor\frac{d-1}{2}\right\rfloor$, then $c=0$.

Proof Consider the basis of $\left(\frac{\Theta}{\partial_{x} \Theta}\right)_{d}^{3}$ given in Lemma 2, and the basis

$$
\theta^{d} \theta^{0}, \theta^{d-1} \theta^{1}, \theta^{d-2} \theta^{2}, \ldots
$$

of $\Theta_{d}^{2}$. For this choice of bases, the map $\frac{\delta}{\delta \theta}$ has a two-step triangular structure. In order to explain that, let us consider the two cases of odd and even $d$ separately.

Consider first the $d=2 k+1$ case. One can check $^{2}$ that the variational derivative $\frac{\delta}{\delta \theta}$ of a basis element $\theta^{k-l+1} \theta^{k-l} \theta^{2 l}$, with $3 l<k$, is equal to

$$
2(-1)^{k-l+1} \theta^{d-2 l} \theta^{2 l}+(d-2 l)(-1)^{k-l+1} \theta^{d-2 l-1} \theta^{2 l+1}
$$

plus terms which are of lower lexicographic order. Notice that the coefficients of the two monomials above are non-vanishing.

Observe that $\frac{\delta}{\delta \theta} \theta^{k+1} \theta^{k} \theta^{0}$ contains the monomials $\theta^{d} \theta^{0}$ and $\theta^{d-1} \theta^{1}$, while the variational derivatives of all other basis elements with $l \geqslant 1$ cannot contain $\theta^{d} \theta^{0}$ and $\theta^{d-1} \theta^{1}$. Thus, if $\frac{\delta \chi}{\delta \theta}=c \cdot \theta^{i} \theta^{d-i}$ for some $i$, then the coefficient of $\theta^{k+1} \theta^{k} \theta^{0}$ in $\chi$ has to be equal to zero.

We can continue this process by induction. Assume that we have already proved that the first $l$ elements of the basis cannot appear in $\chi$. Then the variational derivative of the basis element $\theta^{k-l+1} \theta^{k-l} \theta^{2 l}$ is the only one that contains $\theta^{d-2 l} \theta^{2 l}$ and $\theta^{d-2 l-1} \theta^{2 l+1}$. It follows from the same reason as above, that such basis element cannot appear in $\chi$.

\footnotetext{
${ }^{2}$ Note that the computation is slightly different in the case $3 l=k-1$.
} 
In the case $d=2 k$, we can apply the same reasoning. In this case, the variational derivative $\frac{\delta}{\delta \theta}$ of a basis element $\theta^{k-l} \theta^{k-l-1} \theta^{2 l+1}$, with $3 l<k-2$, is equal to

$$
2(-1)^{k-l} \theta^{d-2 l-1} \theta^{2 l+1}+(d-2 l-1)(-1)^{k-l} \theta^{d-2 l-2} \theta^{2 l+2}
$$

plus terms of lower lexicographic order. Notice that $\theta^{d} \theta^{0}$ never enters the image of any basis element in $\Theta_{d}^{3} / \partial_{x} \Theta_{d-1}^{3}$. Since the coefficients of the two monomials above are non-vanishing, we can apply the same argument as in the case of odd $d$, mutatis mutandis.

Now let us consider an arbitrary element $\chi \in \Theta_{d}^{3}$, such that $\left(\frac{\delta \chi}{\delta \theta}\right)^{2}$ belongs to the image of $\partial_{x}$. From Lemma 5, it follows that $\frac{\delta \chi}{\delta \theta}=c \cdot \theta^{i} \theta^{d-i}$ for some $i=$ $0,1, \ldots,\lfloor d / 2\rfloor$. Then Lemma 6 implies that $\frac{\delta \chi}{\delta \theta}=0$; hence, $\chi$ belongs to the image of $\partial_{x}$.

We have proved that $\chi=0$ as element of $\left(\frac{\Theta}{\partial_{x} \Theta}\right)_{d}^{3}$. Lemma 4 is proved.

\section{The numerical invariants of the Poisson bracket}

In principle all the numerical invariants of a Poisson bracket of the form (1), namely the sequence $\left(c_{1}, c_{2}, \ldots\right)$, can be extracted iteratively solving order by order for the Miura transformation which eliminates the coboundary terms. Providing a general formula for the invariants of a Poisson bivector is hard, since the elimination of each coboundary term affects in principle all the higher-order ones and it is necessary to give an explicit form for the Miura transformation. However, the lowest invariants can be computed as follows.

Proposition 3 Consider a Poisson bracket of the form

$$
\begin{aligned}
& \left\{u\left(x^{1}, x^{2}\right), u\left(y^{1}, y^{2}\right)\right\} \\
& =\left\{u\left(x^{1}, x^{2}\right), u\left(y^{1}, y^{2}\right)\right\}^{0} \\
& \quad+\sum_{k>0} \epsilon^{k} \sum_{\substack{k_{1}, k_{2} \geqslant 0 \\
k_{1}+k_{2} \leqslant k+1}} A_{k ; k_{1}, k_{2}}(u(x)) \delta^{\left(k_{1}\right)}\left(x^{1}-y^{1}\right) \delta^{\left(k_{2}\right)}\left(x^{2}-y^{2}\right),
\end{aligned}
$$

as in (1). Here $A_{k ; k_{1}, k_{2}} \in \mathcal{A}$ and $\operatorname{deg} A_{k ; k_{1}, k_{2}}=k-k_{1}-k_{2}+1$. Then the first numerical invariants of the bracket, giving the normal form of Theorem 1, are

$$
\begin{aligned}
& c_{1}=A_{2 ; 3,0}, \\
& c_{2}=A_{4 ; 5,0}(u)-A_{2 ; 3,0} A_{2 ; 2,1}(u) .
\end{aligned}
$$

Notice that $A_{2 ; 3,0}$ is implied to be a constant. 
Proof We recall that, given a Poisson bracket $P$ of form (1), it can be expanded according to its differential order. For notational compactness, we will denote

$$
P_{k+1}:=\sum_{\substack{k_{1}, k_{2} \geqslant 0 \\ k_{1}+k_{2} \leqslant k+1}} A_{k ; k_{1}, k_{2}}(u(x)) \delta^{\left(k_{1}\right)}\left(x^{1}-y^{1}\right) \delta^{\left(k_{2}\right)}\left(x^{2}-y^{2}\right)
$$

for $k>0$, so that $\operatorname{deg} P_{k}=k$.

In this proof, we replace $\left(x^{1}, x^{2}\right)$ with $(x, y)$ as we did in the previous sections; moreover, with a slight abuse of notation we identify the Dirac's delta derivatives with the corresponding elements of $\hat{\mathcal{F}}$ previously used

$$
\mathfrak{p}_{1}:=\delta\left(x^{1}-y^{1}\right) \delta^{(1)}\left(x^{2}-y^{2}\right) \quad \mathfrak{p}_{k}:=\delta^{(k)}\left(x^{1}-y^{1}\right) \delta\left(x^{2}-y^{2}\right)
$$

Using this notation, the Schouten identity $[P, P]=0$ reads

$$
2\left[\mathfrak{p}_{1}, P_{k}\right]+\sum_{l=2}^{k-1}\left[P_{l}, P_{k-l+1}\right]=0
$$

for $k \geqslant 2$. The first equation is $\left[\mathfrak{p}_{1}, P_{2}\right]=0$; we solved it in [2], finding for $P_{2}$

$$
\begin{array}{lll}
A_{1 ; 2,0}=0 & A_{1 ; 1,1}=0 & A_{1 ; 0,2}=0 \\
A_{1 ; 1,0}=-f(u) \partial_{y} u & A_{1 ; 0,1}=f(u) \partial_{x} u & A_{1 ; 0,0}=0
\end{array}
$$

for any function $f(u)$. Since $H_{2}^{2}(\hat{\mathcal{F}})=0$, we have $P_{2}=\left[X_{1}, \mathfrak{p}_{1}\right]$ and the Miura transformation that eliminates $P_{2}$ from $P$ is $e^{-\operatorname{ad}_{\epsilon X_{1}}}$. The evolutionary vector field $X_{1}$ has characteristic

$$
X_{1}(u)=F(u) \partial_{x} u
$$

where $F(u)=\int^{u} f(s) d s$. We also observe that $\operatorname{ad}_{X_{1}}^{m} \mathfrak{p}_{1}=0$ for $m>1$.

We apply the Miura transformation generated by $-\epsilon X_{1}$ to $P$ and get

$$
\begin{aligned}
\tilde{P}=e^{-\operatorname{ad}_{\epsilon X_{1}} P=} & \mathfrak{p}_{1}+\epsilon^{2} P_{3}+\epsilon^{3}\left(P_{4}-\left[X_{1}, P_{3}\right]\right) \\
& +\epsilon^{4}\left(P_{5}-\left[X_{1}, P_{4}\right]+\frac{1}{2}\left[X_{1},\left[X_{1}, P_{3}\right]\right]\right)+\cdots
\end{aligned}
$$

The first equation of the system (26) for $\tilde{P}$, and the results used in the proof of Lemma 2 give us $P_{3}=c_{1} \mathfrak{p}_{3}+\left[X_{2}, \mathfrak{p}_{1}\right]$. 
$\left[X_{2}, \mathfrak{p}_{1}\right]$ is a bivector whose degree in the number of derivatives w.r.t. $x^{2}$ is at least 1 ; notice that $x^{1}$ corresponds to $x$ and $x^{2}$ corresponds to $y$, in the notation of Sects. 3 and 4 . Hence, we can write

$$
\begin{aligned}
P_{3}= & A_{2 ; 3,0}(u) \mathfrak{p}_{3}+A_{2 ; 2,1}(u) \delta^{(2)}\left(x^{1}-y^{1}\right) \delta^{(1)}\left(x^{2}-y^{2}\right) \\
& +A_{2 ; 1,2}(u) \delta^{(1)}\left(x^{1}-y^{1}\right) \delta^{(2)}\left(x^{2}-y^{2}\right)+A_{2 ; 0,3}(u) \delta\left(x^{1}-y^{1}\right) \delta^{(3)}\left(x^{2}-y^{2}\right) \\
& +\cdots \\
= & c_{1} \mathfrak{p}_{3}+\left[X_{2}, \mathfrak{p}_{1}\right]
\end{aligned}
$$

This equation immediately gives $A_{2 ; 3,0}(u)=A_{2 ; 3,0}=c_{1}$ as in (24). Moreover, we can solve it for $X_{2}$; the characteristic of the evolutionary vector field which is a differential polynomial with top degree w.r.t. the $x$ derivatives is $1 / 2 A_{2 ; 2,1}(u) \partial_{x}^{2} u+\tilde{A}(u)\left(\partial_{x} u\right)^{2}$. Here we are interested only in first summand because it is the one that gives the highest number of $x$-derivatives in $\left[X_{2}, \mathfrak{p}_{r}\right]$, for any $r$.

We apply to $\tilde{P}$ the Miura transformation $e^{-\operatorname{ad}_{\epsilon^{2} X_{2}}}$ to eliminate the coboundary term of $P_{3}$ and are left with

$$
\begin{aligned}
e^{-\mathrm{ad}_{\epsilon^{2} X_{2}} \tilde{P}=} & \mathfrak{p}_{1}+\epsilon^{2} c_{1} \mathfrak{p}_{3}+\epsilon^{3}\left(P_{4}-c_{1}\left[X_{1}, \mathfrak{p}_{3}\right]-\left[X_{1},\left[X_{2}, \mathfrak{p}_{1}\right]\right]\right) \\
& +\epsilon^{4}\left(P_{5}-\left[X_{1}, P_{4}\right]+\frac{1}{2} c_{1}\left[X_{1},\left[X_{1}, \mathfrak{p}_{3}\right]\right]+\frac{1}{2}\left[X_{1},\left[X_{1},\left[X_{2}, \mathfrak{p}_{1}\right]\right]\right]\right. \\
& \left.-c_{1}\left[X_{2}, \mathfrak{p}_{3}\right]-\frac{1}{2}\left[X_{2},\left[X_{2}, \mathfrak{p}_{1}\right]\right]\right)+\cdots
\end{aligned}
$$

We now use the fact that $H_{4}^{2}(\hat{\mathcal{F}})=0$ to get

$$
P_{4}=c_{1}\left[X_{1}, \mathfrak{p}_{3}\right]+\left[X_{1},\left[X_{2}, \mathfrak{p}_{1}\right]\right]+\left[X_{3}, \mathfrak{p}_{1}\right]
$$

for some homogeneous vector field $X_{3}$ of degree 3. This allows us to replace $P_{4}$ in (27) and to apply the Miura transform $e^{-\operatorname{ad}_{\epsilon^{3} X_{3}}}$ to it to get rid of the term $\epsilon^{3}$ in the expansion. The terms of order $<3$ are left unaffected by this transformation, while the coefficient of $\epsilon^{4}$ becomes

$$
\begin{gathered}
P_{5}-\left[X_{1},\left[X_{3}, \mathfrak{p}_{1}\right]\right]-\frac{1}{2} c_{1}\left[X_{1},\left[X_{1}, \mathfrak{p}_{3}\right]\right]-\frac{1}{2}\left[X_{1},\left[X_{1},\left[X_{2}, \mathfrak{p}_{1}\right]\right]\right] \\
-c_{1}\left[X_{2}, \mathfrak{p}_{3}\right]-\frac{1}{2}\left[X_{2},\left[X_{2}, \mathfrak{p}_{1}\right]\right]=c_{2} \mathfrak{p}_{5}+\left[X_{4}, \mathfrak{p}_{1}\right]
\end{gathered}
$$

where the equality is given by our results about $H_{5}^{2}(\hat{\mathcal{F}})$ and the proof of Lemma 2 . The invariant $c_{2}$ must be read taking the coefficient of $\mathfrak{p}_{5}$ in the lefthand side of the equation: this coefficient cannot be obtained by summands that are of $y$-degree bigger or equal to 1 . Thus we focus on the summands

$$
P_{5}-\frac{1}{2}\left[X_{1},\left[X_{1}, \mathfrak{p}_{3}\right]\right]-c_{1}\left[X_{2}, \mathfrak{p}_{3}\right]=c_{2} \mathfrak{p}_{5}+\cdots
$$


A direct computation shows that in $\operatorname{ad}_{X_{1}}^{2} \mathfrak{p}_{3}$ the term $\mathfrak{p}_{5}$ does not appear, while it does appear in $\left[X_{2}, \mathfrak{p}_{3}\right]$. Using the form of $X_{2}$ we have previously derived, we find

$$
P_{5}=\left(A_{4 ; 5,0}(u) \mathfrak{p}_{5}+\cdots\right)=\left(c_{2}+c_{1} A_{2 ; 2,1}(u)\right) \mathfrak{p}_{5}+\cdots
$$

from which we get (25).

Example 2 We can compute all the numerical invariants when the Poisson bracket is particularly simple. Let us consider the bracket

$$
\begin{aligned}
\{u(x), u(y)\}= & \delta\left(x^{1}-y^{1}\right) \delta^{\prime}\left(x^{2}-y^{2}\right)+\delta^{\prime \prime \prime}\left(x^{1}-y^{1}\right) \delta\left(x^{2}-y^{2}\right) \\
& +\delta^{\prime \prime}\left(x^{1}-y^{1}\right) \delta^{\prime}\left(x^{2}-y^{2}\right) .
\end{aligned}
$$

Proposition 3 immediately tells us that $c_{1}=1$ and $c_{2}=-1$. Let us denote for brevity $\mathfrak{p}_{s, t}$ the bivector corresponding to $\frac{1}{2} \int \theta \theta^{(s, t)}$. The bivector corresponding to the bracket then reads $P=\mathfrak{p}_{1}+\mathfrak{p}_{3}+\mathfrak{p}_{2,1}$, and $\mathfrak{p}_{2,1}=\operatorname{ad}_{X_{2}} \mathfrak{p}_{1}$. It is very easy to derive $X_{2}=\frac{1}{2} u_{2 x} \theta$. We have $\operatorname{ad}_{X_{2}} \mathfrak{p}_{s, t}=\mathfrak{p}_{s+2, t}$. The Miura transformation $e^{-\operatorname{ad}_{X_{2}}}$ applied to $P$ gives

$$
\begin{aligned}
P_{(1)} & =\mathfrak{p}_{1}+\sum_{n=0}^{\infty} \frac{(-1)^{n}}{n !} \operatorname{ad}_{X_{2}}^{n} \mathfrak{p}_{3}+\sum_{n=1}^{\infty}(-1)^{n}\left(\frac{1}{n !}-\frac{1}{(n+1) !}\right) \operatorname{ad}_{X_{2}}^{n+1} \mathfrak{p}_{1} \\
& =\mathfrak{p}_{1}+\sum_{n=0}^{\infty} \frac{(-1)^{n}}{n !} \mathfrak{p}_{3+2 n}+\sum_{n=1}^{\infty}(-1)^{n}\left(\frac{1}{n !}-\frac{1}{(n+1) !}\right) \mathfrak{p}_{2 n+2,1}
\end{aligned}
$$

Notice that the term $n=0$ in the first sum gives the only contribution of order 3 , giving $c_{1}=1$. The further $\mathfrak{p}_{1}$-coboundary term should be read in the $n=1$ term of the second sum, namely for $-\frac{1}{2} \mathfrak{p}_{4,1}=\operatorname{ad}_{X_{4}} \mathfrak{p}_{1}$. The next Miura transformation leads to

$$
\begin{aligned}
P_{(2)}= & \mathfrak{p}_{1}+\sum_{m=0}^{\infty} \sum_{n=0}^{\infty} \frac{(-1)^{n+2 m}}{2^{m} m ! n !} \mathfrak{p}_{2 n+4 m+3}+\sum_{m=1}^{\infty} \frac{(-1)^{2 m}}{2^{m} m !} \mathfrak{p}_{4 m, 1} \\
& +\sum_{m=0}^{\infty} \sum_{n=2}^{\infty}\left(\frac{1}{n !}-\frac{1}{(n-1) !}\right) \frac{(-1)^{n+2 m}}{2^{m} m ! n !} \mathfrak{p}_{2 n+4 m, 1} .
\end{aligned}
$$

The procedure goes on-always requiring us to find the vector field cancelling the lowest order term of the form $\mathfrak{p}_{s, 1}$. At each step, we will need vector fields $X_{2 s+2}$ such that

$$
\operatorname{ad}_{X_{2 s}} \mathfrak{p}_{1}=\frac{(-1)^{s+1}}{s} \mathfrak{p}_{2 s, 1}
$$


and we obtain

$$
P(\infty)=\left(\prod_{s=1 \ldots \infty}^{\curvearrowleft} e^{-\mathrm{ad}_{2 s}}\right) P
$$

The Miura transformation cancels all the terms of the form $\mathfrak{p}_{s, 1}$ and we are left with the following expression for the Poisson bivector brought to the normal form:

$$
P_{(\infty)}=\mathfrak{p}_{1}+\sum_{m_{1}, m_{2}, \ldots=0}^{\infty} \frac{(-1)^{m_{1}+2 m_{2}+3 m_{3}+\cdots}}{m_{1} ! m_{2} ! m_{3} ! \cdots 2^{m_{2}} 3^{m_{3}} \cdots} \mathfrak{p}_{3+2 m_{1}+4 m_{2}+6 m_{3}+\cdots}
$$

We recall that $\frac{1}{2} \int \theta \partial_{x}^{k} \theta=\mathfrak{p}_{k}$. Hence, the infinite sum can be seen as a series expansion for $\frac{1}{2} \int \theta \partial_{x}^{3} /\left(1+\partial_{x}^{2}\right) \theta$ as follows:

$$
\begin{gathered}
\frac{1}{2} \int \theta \partial_{x}^{3}\left(\sum_{m_{1}=0}^{\infty} \frac{(-1)^{m_{1}}}{m_{1} !} \partial_{x}^{2 m_{1}}\right)\left(\sum_{m_{2}=0}^{\infty} \frac{(-1)^{2 m_{2}}}{2^{m_{2}} m_{2} !} \partial_{x}^{4 m_{2}}\right)\left(\sum_{m_{3}=0}^{\infty} \frac{(-1)^{3 m_{3}}}{3^{m_{2}} m_{3} !} \partial_{x}^{6 m_{3}}\right) \cdots \theta \\
=\frac{1}{2} \int \theta\left(\partial_{x}^{3} e^{-\partial_{x}^{2}+\frac{\partial_{x}^{4}}{2}-\frac{\partial_{x}^{6}}{3}+\cdots}\right) \theta=\frac{1}{2} \int \theta \partial_{x}^{3} e^{-\log \left(1+\partial_{x}^{2}\right)} \theta=\frac{1}{2} \int \theta \frac{\partial_{x}^{3}}{1+\partial_{x}^{2}} \theta .
\end{gathered}
$$

We stress the fact that all these identities should always been understood in terms of formal power expansion. On the other hand, a more obvious expansion for the same expression is

$$
\frac{1}{2} \int \theta \frac{\partial_{x}^{3}}{1+\partial_{x}^{2}} \theta=\frac{1}{2} \int \theta \partial_{x}^{3} \sum_{k=0}^{\infty}(-1)^{k} \partial_{x}^{2 k} \theta
$$

that translates into

$$
P_{(\infty)}=\mathfrak{p}_{1}-\sum_{k=1}^{\infty}(-1)^{k} \mathfrak{p}_{2 k+1}
$$

and gives us all the numerical invariants of (28).

Example 3 (Hamiltonian structure of Helmoltz's equation) Helmoltz's equation describes the time evolution of the vorticity in an ideal incompressible fluid [17]. In the two-dimensional case, it is an evolutionary equation for the scalar vorticity of the fluid $\omega(x, y)$ with velocity field $\mathbf{v}(x, y)=(u(x, y), v(x, y))$, given by

$$
\omega_{t}=-u \omega_{x}-v \omega_{y}
$$

The vorticity of the fluid is the scalar quantity $\omega=v_{x}-u_{y}$. Such an equation is not integrable, but it is Hamiltonian [23] with respect to the functional 


$$
H=\frac{1}{2} \int\left(u^{2}+v^{2}\right)
$$

and the Poisson bracket

$$
\{\omega(x, y), \omega(w, z)\}=\omega_{x} \delta(x-w) \delta^{(1)}(y-z)-\omega_{y} \delta^{(1)}(x-w) \delta(y-z)
$$

It should be noticed that also in this case, as for Example 1, the Hamiltonian functional is not local in the field $\omega$. Indeed, the incompressibility of the fluid in two dimensions allows us to introduce the stream function $\psi(x, y)$ such that $u=\psi_{y}$ and $v=-\psi_{x}$, for which $\Delta \psi=-\omega$ and $\delta H / \delta \omega=-\psi$.

The bracket (29) is not of the form (1); however, it is compatible with the bracket $\{\omega(x, y), \omega(w, z)\}^{0}=\delta(x-w) \delta^{(1)}(y-z)$, which allows us to consider the first-order deformation given by the Poisson bivector

$$
P=\mathfrak{p}_{1}+\frac{1}{2} \int\left(u_{x} \theta \theta^{(0,1)}-u_{y} \theta \theta^{(1,0)}\right) .
$$

The deformation is a coboundary in the Poisson cohomology of $\mathfrak{p}_{1}$, which follows from $H_{2}^{2}\left(\mathfrak{p}_{1}\right)=0$. In particular, it is obtained by $\left[\mathfrak{p}_{1}, X_{1}\right]$ with $X_{1}=-u u_{x} \theta$. Moreover, a simple computation shows that $\left[X_{1},\left[X_{1}, \mathfrak{p}_{1}\right]\right]=0$. This means that with a Miura transformation $\exp ^{-X_{1}} P=\mathfrak{p}_{1}$, and the normal form of the bracket (30) has numerical invariants $c_{k} \equiv 0$.

Acknowledgements We would like to thank Jenya Ferapontov for several useful observations and Dario Merzi for suggesting a clever identity in Example 2. M. C. was supported by the INdAM-COFUND-2012 Marie Curie fellowship "MPoisCoho-Poisson cohomology of multidimensional Hamiltonian operators".

Open Access This article is distributed under the terms of the Creative Commons Attribution 4.0 International License (http://creativecommons.org/licenses/by/4.0/), which permits unrestricted use, distribution, and reproduction in any medium, provided you give appropriate credit to the original author(s) and the source, provide a link to the Creative Commons license, and indicate if changes were made.

\section{References}

1. Barakat, A.: On the moduli space of deformations of bihamiltonian hierarchies of hydrodynamic type. Adv. Math. 219(2), 604-632 (2008)

2. Carlet, G., Casati, M., Shadrin, S.: Poisson cohomology of scalar multidimensional Dubrovin-Novikov brackets. J. Geom. Phys. 114, 404-419 (2017)

3. Carlet, G., Kramer, R., Shadrin, S.: Central invariants revisited. arXiv:1611.09134

4. Carlet, G., Posthuma, H., Shadrin, S.: Bihamiltonian cohomology of $K \mathrm{~d} V$ brackets. Commun. Math. Phys. 341(3), 805-819 (2016)

5. Carlet, G., Posthuma, H., Shadrin, S.: The bi-Hamiltonian cohomology of a scalar Poisson pencil. Bull. Lond. Math. Soc. 48(4), 617-627 (2016)

6. Carlet, G., Posthuma, H., Shadrin, S.: Deformations of semisimple Poisson pencils of hydrodynamic type are unobstructed. arXiv:1501.04295

7. Casati, M.: On deformations of multidimensional Poisson brackets of hydrodynamic type. Commun. Math. Phys. 335(2), 851-894 (2015)

8. Case, K.M.: A theorem about Hamiltonian systems. Proc. Natl. Acad. Sci. USA 81(18), 5893-5895 (1984) 
9. Degiovanni, L., Magri, F., Sciacca, V.: On deformation of Poisson manifolds of hydrodynamic type. Commun. Math. Phys. 253(1), 1-24 (2005)

10. Dubrovin, B.A., Liu, S.-Q., Zhang, Y.: On Hamiltonian perturbations of hyperbolic systems of conservation laws. I. Quasi-triviality of bi-Hamiltonian perturbations. Commun. Pure Appl. Math. 59(4), 559-615 (2006)

11. Dubrovin, B.A., Liu, S.-Q., Zhang, Y.: Frobenius manifolds and central invariants for the DrinfeldSokolov biHamiltonian structures. Adv. Math. 219(3), 780-837 (2008)

12. Dubrovin, B.A., Novikov, S.P.: Hamiltonian formalism of one-dimensional systems of the hydrodynamic type and the Bogolyubov-Whitham averaging method (Russian). Dokl. Akad. Nauk SSSR 270(4), 781-785 (1983) (transl: Soviet Math. Dokl. 27, 665-669 (1983))

13. Dubrovin, B.A., Novikov, S.P.: Poisson brackets of hydrodynamic type (Russian). Dokl. Akad. Nauk SSSR 279(2), 294-297 (1984) (transl: Soviet Math. Dokl. 30, 6516-6554 (1984))

14. Dubrovin, B.A., Zhang, Y.: Normal forms of hierarchies of integrable PDEs, Frobenius manifolds and Gromov-Witten invariants. arXiv:math/0108160

15. Ferapontov, E.V., Lorenzoni, P., Savoldi, A.: Hamiltonian operators of Dubrovin-Novikov type in 2D. Lett. Math. Phys. 105(3), 341-377 (2015)

16. Getzler, E.: A Darboux theorem for Hamiltonian operators in the formal calculus of variations. Duke Math. J. 111(3), 535-560 (2002)

17. Lamb, H.: Hydrodynamics. Cambridge University Press, Cambridge (1932)

18. Liu, S.-Q., Zhang, Y.: Jacobi structures of evolutionary partial differential equations. Adv. Math. 227(1), 73-130 (2011)

19. Liu, S.-Q., Zhang, Y.: Bihamiltonian cohomologies and integrable hierarchies I: a special case. Commun. Math. Phys. 324(3), 897-935 (2013)

20. Lorenzoni, P.: Deformations of bi-Hamiltonian structures of hydrodynamic type. J. Geom. Phys. 44(23), 331-375 (2002)

21. Mokhov, O.I.: Poisson brackets of Dubrovin-Novikov type (DN-brackets) (Russian). Funktsional. Anal. i Prilozhen 22(4), 92-93 (1988) (transl: Funct. Anal. Appl. 22(4), 336-338 (1989))

22. Mokhov, O.I. Classification of nonsingular multidimensional Dubrovin-Novikov brackets (Russian). Funktsional. Anal. i Prilozhen 42(1), 39-52, 95-96 (2008) (transl: Funct. Anal. Appl. 42(1), 33-44 (2008))

23. Olver, P.J.: A nonlinear Hamiltonian structure for the Euler equations. J. Math. Anal. Appl. 89(1), 233-250 (1982)

24. Zakharov, V.E., Shabat, A.B.: A plan for integrating the nonlinear equations of mathematical physics by the method of the inverse scattering problem I (Russian). Funkc. Anal. i Prilozhen 8(3), 43-53 (1974) 